\title{
Atomic Scale Near-Edge Structures of a Structurally Abrupt Ni-SrTiO Interface $_{3}$
}

Guillaume Radtke ${ }^{1}$, Matthieu Bugnet ${ }^{2}$, Quentin M. Ramasse ${ }^{3,4}$, Marcel Hennes ${ }^{5}$, Xiaorong Weng ${ }^{5}$, Dominique Demaille ${ }^{5}$, Yunlin Zheng ${ }^{5}$ and Franck Vidal ${ }^{5}$

1. Sorbonne Université, CNRS, IMPMC, Paris, France.

2. Univ Lyon, INSA Lyon, UCBL Lyon1, MATEIS, CNRS UMR 5510, Villeurbanne, France.

3. SuperSTEM Laboratory, SciTech Daresbury Campus, Daresbury, United Kingdom.

4. School of Physics and School of Chemical and Process Engineering, University of Leeds, Leeds, United Kingdom.

5. Sorbonne Université, CNRS, INSP, Paris, France.

The growth of three dimensional spintronic structures has been proposed as a promising route to overcome the limits of current data storage technologies. Among the potential candidates, selfassembled ferromagnetic nanowires (NWs) embedded in an insulating matrix have recently attracted considerable attention (e.g. Ni in $\mathrm{SrTiO}_{3}$, see Fig. 1). This interest is mainly motivated by the possibility to tune the magnetic anisotropy either through the control of the chemical composition of the NWs or through the presence of interfacially-induced strain giving rise to magneto-elastic anisotropy [1]. In particular, the detection of Ni oxidation, which would be highly detrimental, via the potential presence of a NiO layer between the NW and the matrix, is of interest. This is achieved by investigating the Ni$\mathrm{L}_{23}$ near-edge structures (ELNES), which show substantial differences between metallic $\mathrm{Ni}$ and $\mathrm{Ni}^{2+}[2]$, and the O-K and $\mathrm{Ti}_{-} \mathrm{L}_{23}$ ELNES from the matrix to the $\mathrm{Ni}_{\mathrm{SrTiO}}$ interface [3]. In this work, the local chemistry of the $\mathrm{Ni}: \mathrm{SrTiO}_{3}$ interface is probed at the atomic scale in the aberration-corrected scanning transmission electron microscope (STEM). High energy resolution for ELNES ( 160 meV), and sub- $\AA$ spatial resolution were used in a monochromated Cs-corrected Nion HERMES, operating at $60 \mathrm{kV}$.

The main results are summarized in Fig. 2. The series of spectra extracted from region $\mathrm{P} 1$, on a $\mathrm{TiO}_{2}$ atomic plane located one matrix unit cell $(\sim 3.9 \AA)$ from the interface, still displays all the characteristic features of the bulk electronic structure of $\mathrm{SrTiO}_{3}$ without any detectable Ni. Spectra from region P2, located on the last atomic plane of the matrix from the HAADF contrast, are largely modified. The symmetry breaking induced by the presence of the interface, and the pronounced covalent character of the bonding with neighboring $\mathrm{Ni}$ atoms lead to strong broadening of the Ti-L $\mathrm{L}_{23}$ ELNES, where the $\mathrm{t}_{2 \mathrm{~g}} \mathrm{e}_{\mathrm{g}}$ splitting is barely resolved. The O-K ELNES still exhibits the signature of hybridization with Ti-3d states through the presence of a sharp peak $a$, and a broader structure $b$. Interestingly, the missing SrO plane on the left side of the interface leads to a considerable loss of spectral weight in the region of the double structure $c$, primarily associated with the Sr- $4 d$ empty states. Although a weak Ni signal is already detected in this region, which might be partly related to the delocalization inherent to atomicresolved EELS, the presence of $\mathrm{Ni}$ in the last atomic planes of the matrix cannot be ruled out. The modification of the Ti-L $\mathrm{L}_{23}$ ELNES is particularly abrupt along this [100]STO $\|[100] \mathrm{Ni}$ interface, compared to the $[110] \mathrm{STO} \|[110] \mathrm{Ni}$ facets. Region P3 corresponds to the first atomic plane that can be considered structurally part of the nanowire from the HAADF contrast. The Ti- $\mathrm{L}_{23}$ edge in this area is also further broadened to reach a shape that closely resembles that of the Ti- $\mathrm{L}_{23}$ edge in bulk Ti or NiTi alloy. In region $\mathrm{P} 4$, the Ni- $\mathrm{L}_{23}$ edge is essentially that of bulk $\mathrm{Ni}$, while very weak $\mathrm{Ti}$ and $\mathrm{O}$ signals are still detectable. Finally, regions P5 and P6 are characteristic of pure bulk Ni. The Ni- $\mathrm{L}_{3} \mathrm{to}_{2}$ ratios show no substantial deviation from the bulk Ni value in this series of spectra A-E. This is consistent with the $\mathrm{O}-\mathrm{K}$ ELNES at the interface, which does not display any characteristic features of NiO. 
In conclusion, spectroscopic investigations of the structurally abrupt $\mathrm{Ni}: \mathrm{SrTiO}_{3}$ interface at the atomicscale indicate the presence of a weak interdiffusion over a few atomic planes only [3]. This leads essentially to a Ni metallization of the last $\mathrm{TiO}_{\mathrm{x}}(\mathrm{x}<2)$ atomic plane of the matrix. These results demonstrate the absence of Ni oxidation. This establishes Ni:STO as a model system for the study of strain engineering of the magnetic anisotropy in vertically aligned nanocomposites [4].

\section{References:}

[1] V Schuler et al., Nanotechnology 27 (2016), p. 495601.

[2] RD Leapman et al., Phys. Rev. B 26 (1982), p. 614.

[3] G Radtke et al., submitted (2019).

[4] STEM-EELS experiments were carried out at SuperSTEM, the EPSRC National Research Facility for Advanced Electron Microscopy. This work was supported by French state funds managed by the ANR within the Investissements d'Avenir programme (ANR-11-IDEX-0004-02), within the framework of the Cluster of Excellence MATISSE led by Sorbonne Universités. M. Hennes acknowledges financial support from the French Embassy in Berlin (Service pour la Science et la Technologie) and Campus France. X. Weng acknowledges financial support from China Scholarship Council (CSC).
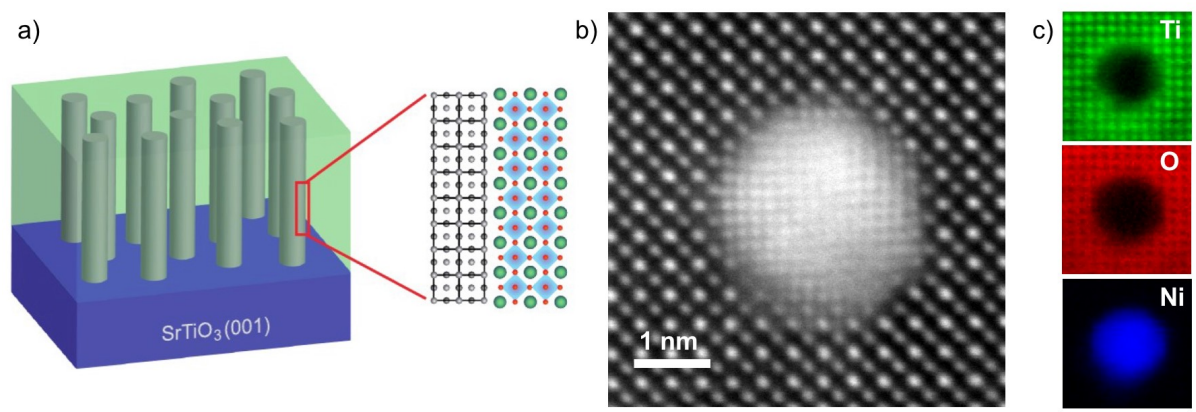

Figure 1. a) Sketch of a $\mathrm{Ni}: \mathrm{SrTiO}_{3} / \mathrm{SrTiO}_{3} \quad$ vertically aligned nanostructures, where $\mathrm{Ni}$ NWs are epitaxially grown on $\mathrm{SrTiO}_{3}(001)$ substrate, and embedded within an $\mathrm{SrTiO}_{3}$ matrix (green). b) HAADF inplane view of a single NW, and c) elemental EELS maps of a NW.
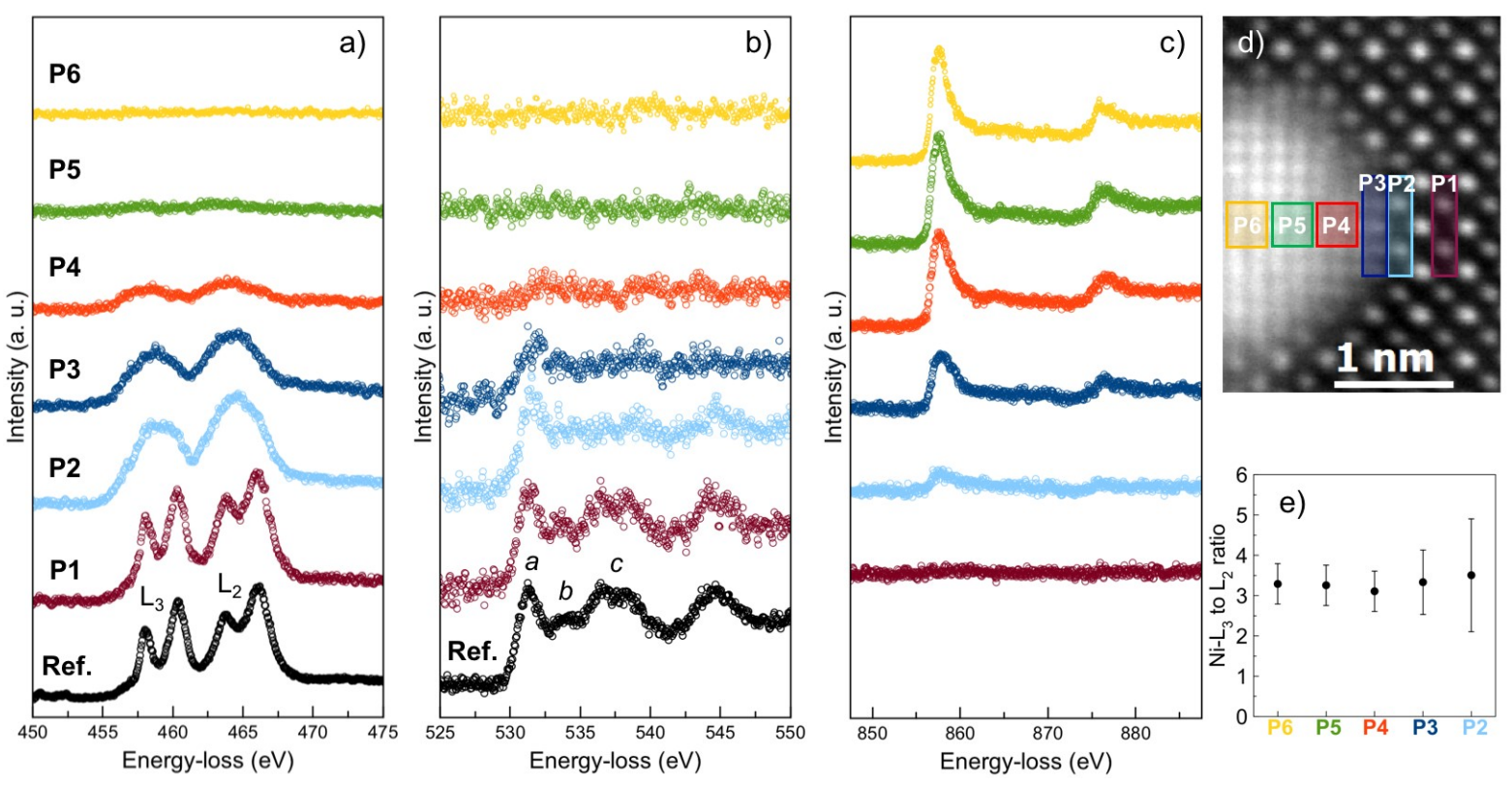

Figure 2. ELNES variations of the a) $\mathrm{Ti}_{-}-\mathrm{L}_{23}$, b) $\mathrm{O}-\mathrm{K}$, and c) $\mathrm{Ni}-\mathrm{L}_{23}$ edges across the Ni:STO interface in d). The $\mathrm{Ni} \mathrm{L}_{3} / \mathrm{L}_{2}$ intensity ratio in e) shows no variations, supporting the absence of $\mathrm{Ni}^{2+}$. 